\title{
Perfect state transfer on hypercubes and its implementation using superconducting qubits
}

\author{
Siddhant Singh $\odot,{ }^{1, *}$ Bibhas Adhikari $\odot, 2,3, \uparrow$ Supriyo Dutta $\odot,{ }^{3, \sharp}$ and David Zueco ${ }^{4, \S}$ \\ ${ }^{1}$ Department of Physics, Indian Institute of Technology Kharagpur, Kharagpur 721302, India \\ ${ }^{2}$ Department of Mathematics, Indian Institute of Technology Kharagpur, Kharagpur 721302, India \\ ${ }^{3}$ Center for Theoretical Studies, Indian Institute of Technology Kharagpur, Kharagpur 721302, India \\ ${ }^{4}$ Instituto de Nanociencia y Materiales de Aragón and Departamento de Física de la Materia Condensada, CSIC, \\ Universidad de Zaragoza, Zaragoza 50009, Spain
}

(Received 8 June 2020; revised 7 November 2020; accepted 10 November 2020; published 14 December 2020)

\begin{abstract}
We propose a protocol for perfect state transfer between any pair of vertices in a hypercube. Given a pair of distinct vertices in the hypercube, we determine a subhypercube that contains the pair of vertices as antipodal vertices. Then a switching process is introduced for determining the subhypercube of a memory-enhanced hypercube that facilitates perfect state transfer between the desired pair of vertices. Furthermore, we propose a physical architecture for the pretty good state transfer implementation of our switching protocol with fidelity arbitrary close to unity, using superconducting transmon qubits with tunable couplings. The switching is realized by the control over the effective coupling between the qubits resulting from the effect of ancilla qubit couplers for the graph edges. We also report an error bound on the fidelity of state transfer due to faulty implementation of our protocol.
\end{abstract}

DOI: 10.1103/PhysRevA.102.062609

\section{INTRODUCTION}

Quantum computation often requires the transference of an arbitrary quantum state from one location to another [1]. Especially in large-scale quantum information processing (QIP), this is an important task, connecting two sites that may belong to the same or different quantum processors. The latter is nontrivial for many QIP realizations, such as solid state quantum computing and superconducting quantum computing [2-8]. It is also very important to find the systems that support this quantum information exchange between distant sites to realize this phenomenon. For short-distance communication (such as adjacent quantum processors), methods for interfacing different kinds of physical systems are required, for example, ion traps $[9,10]$, superconducting circuits $[11,12]$, and boson lattices [13]. The task of state transfer is incorporated with the idea of reducing the manipulation required to communicate between distant computational qubits in a large-scale quantum computer $[14,15]$. Scalability of quantum processors is a deep concern in the development of quantum computing hardware [16-18]. This is essential for determining how good an architecture is for quantum information processing [19].

Quantum state transfer with $100 \%$ fidelity is known as perfect state transfer (PST) and this idea using interacting spin-1/2 particles was first proposed in [20]. It was established by utilizing a combinatorial graph structure as a platform for an actual quantum network in the first-excitation

\footnotetext{
*siddhant.singh@iitkgp.ac.in

†bibhas@maths.iitkgp.ac.in

ॠdosupriyo@iitkgp.ac.in

§dzueco@unizar.es
}

subspace of a multiqubit system [21,22]. In general, this involves mixed states of network qubits [23]; however, showing PST for pure states in a graph suffices to prove the phenomenon. Perfect state transfer can be used in entanglement transfer, quantum communication, signal amplification, quantum information recovery, and implementation of universal quantum computation [15,24-26]. It is important to find classes of graphs where PST is possible and equally important to find graphs where it is $[27,28]$, in order to classify how good an architecture is for quantum information processing. The idea of pretty good state transfer is also studied, where the transfer fidelity is less than unity but occurring on a large number of graphs that support state transfer [29-33] and in general these graphs can be weighted [34]. More general graphs such as signed graphs [35] and oriented graphs [36] are also studied. Perfect state transfer for qudits has also been classified for some networks [37,38]. One of the big challenges for scalable quantum architecture is the imperfect two-qubit interaction. For PST with maximum fidelity, the pairwise interaction should be improved for large-scale quantum processors [39]. Different physical systems for quantum computation have different advantages, such as high fidelity and control in ion traps $[10,40]$ versus the scalability of superconducting circuits $[8,41,42]$. Perfect state transfer was demonstrated in the latter, with tunable qubit couplings [43]. Here we propose a protocol for large-scale quantum processors with support on a hypercube network.

The scheme established in [44,45] allows PST over arbitrarily long distances. Here the shortcoming is that the PST is possible only between antipodal vertices. A switching technique was proposed in [46] where in a complete graph $K_{n}$, switching off one link establishes PST in nonadjacent qubits. This enables PST for more vertices but still does not enable 
routing to different vertices and there is no scalability; the graph remains fixed. One attempt at switching and routing was proposed in [47] which involves creating new edges and coupling for qubits but is still not scalable. Various other works describe methods of routing of excitations in spin chains [48-50], limited to one dimension. In this work we resolve this problem through our hypercube switching scheme. The goal of this paper is threefold: (i) showing perfect state transfer is possible between any pair of vertices in an $n$-dimensional hypercube by introducing a concept of switching on and off of edges of the hypercube, (ii) defining an effective Hamiltonian that can implement the subhypercube architecture with the proposed switching, and (iii) finding an error bound of the fidelity of the PST for an inaccurate implementation of the subhypercube in an experimental setup.

The rest of the paper is organized as follows. We recall some preliminary results in Sec. II that are needed to establish our results. In Sec. III, given a pair of distinct vertices in an $n$-dimensional hypercube $\mathcal{Q}_{n}$, a unique subhypercube $\mathcal{Q}_{d}$ $(d \leqslant n)$ is determined such that the given pair of vertices is antipodal vertices of $\mathcal{Q}_{d}$. We also describe how a memoryenhanced hypercube enables one to identify the vertices of the subhypercube. Consequently, the perfect state transfer is established between those pair of vertices. A proposal for the implementation of our switching protocol is presented in Sec. IV using superconducting transmon qubits. We summarize in Sec. V.

\section{PRELIMINARIES}

A graph is an ordered pair $G=(V, E)$, where $V$ denotes the set of vertices (or nodes) and $E \subseteq\{(u, v) \in V \times V \mid u \neq v\}$ denotes the set of edges, which are unordered pairs of vertices. Let $I \subseteq V$. Then a subgraph of the graph $G$ defined by the vertex set $I$ is called an induced subgraph if two distinct vertices in $I$ are linked by an edge in the subgraph if and only if they are linked by an edge in $G$ [51]. Obviously, an induced subgraph defined by a set of vertices is unique.

Let $G$ be a graph with $n$ vertices with $n=|V|$ and $V=$ $\{0,1,2, \ldots, n-1\}$. Then the adjacency matrix $A(G)=\left[a_{i j}\right]$ associated with $G$ is defined as $a_{i j}=1$ if $(i, j) \in E$ and 0 otherwise. Obviously, $A(G)$ is a symmetric matrix of order $n \times n$. The degree of $i \in V$ is defined as $\operatorname{deg}(i)=\sum_{j=0}^{n-1} a_{i j}$. The degree matrix $D(G)=\operatorname{diag}\left(d_{0}, \ldots, d_{n-1}\right)$ of $G$ is a diagonal matrix where $d_{i}=\operatorname{deg}(i), 0 \leqslant i \leqslant n-1$. The graph Laplacian matrix $L(G)$ associated with the graph $G$ is defined as $L(G)=D(G)-A(G)$. These two matrices encode the structure of the graph and determine the architecture by determining qubits' mutual couplings and connectivity.

Perfect state transfer of a state in a many-qubit system is formulated by a combinatorial graph in which the edges of the graph represents couplings of qubits. This is interpreted by beginning with a single-qubit state (generally mixed) $\rho_{\text {qubit }}^{u}$ at some site $u$, with $\rho_{\text {in }}$ taken as the state of the rest of the system. After evolution for some finite time $t_{0}$, with an interaction Hamiltonian $H$, the final evolved state

$$
e^{-i H t_{0} / \hbar}\left(\rho_{\text {qubit }}^{u} \otimes \rho_{\text {in }}\right) e^{i H t_{0} / \hbar}=\rho_{\text {qubit }}^{v} \otimes \rho_{\text {out }}
$$

is obtained, thereby transmitting the respective qubit state to another desired vertex $v$ of the graph. In general, $\rho_{\text {qubit }}$ is a density matrix; however, in this paper we consider that it corresponds to a pure state. The most simplified case for such realization is the one-dimensional chain of qubits.

There are two kinds of well-known interaction Hamiltonians for the pairwise interactions defined by the edges between the qubits which are placed at the vertices of a graph. The first is the $X Y$ model in two spatial degrees of freedom,

$$
\begin{aligned}
\frac{H_{X Y}}{\hbar} & =\sum_{(i, j) \in E(G)} J_{i j}\left(\sigma_{i}^{x} \sigma_{j}^{x}+\sigma_{i}^{y} \sigma_{j}^{y}\right) \\
& =\sum_{(i, j) \in E(G)} 2 J_{i j}\left(\sigma_{i}^{+} \sigma_{j}^{-}+\sigma_{i}^{-} \sigma_{j}^{+}\right),
\end{aligned}
$$

where $\sigma_{i}^{ \pm}$are the ladder operators acting on the qubit placed at vertex $i$ such that $\sigma_{i}^{ \pm}=\sigma_{i}^{x} \pm i \sigma_{i}^{y}$, with $\sigma_{i}^{x, y}$ the Pauli matrices. The second connection is via the three-dimensional Heisenberg model

$$
\frac{H_{H e i}}{\hbar}=-\sum_{(i, j) \in E(G)} J_{i j} \vec{\sigma}_{i} \cdot \vec{\sigma}_{j}+\sum_{j} B_{j} \sigma_{j}^{z},
$$

where $\vec{\sigma}_{i}$ is Pauli matrix vector $\vec{\sigma}_{i}=\left(\sigma_{i}^{x}, \sigma_{i}^{y}, \sigma_{i}^{z}\right)$ for the $i$ th spin and $I_{i}$ is the identity operator for the $i$ th vertex.

In this paper we consider the general many-body $X Y$ coupling Hamiltonian as well as the Heisenberg Hamiltonian and consider the coupling strength $J_{i j}$ to be a real parameter that can be continuously changed. In addition, we consider the local fields $B_{j}$ to tune the diagonal terms of the Hamiltonian such that it coincides with the Laplacian of the graph. This special choice is always possible [44]. We also emphasize that these two coupling Hamiltonians need not necessarily correspond to a spin- $1 / 2$ particle interaction. In the first-excitation subspace for the qubits, the $X Y$-coupling Hamiltonian and Heisenberg Hamiltonian action are equivalent to adjacency and Laplacian action, respectively, for the corresponding graph, in the vertex space [21]. Initially, the system is in its ground state $|0\rangle=|000 \ldots 0\rangle$, where the ket $|0\rangle$ denotes the single-qubit ground state. We have the graph vertex-space states $|i\rangle=|00 \ldots 010 \ldots 0\rangle(i=1,2, \ldots, n)$, in which the qubit at the $i$ th site is in the first-excited state $|1\rangle$. To start the PST procedure, $A$ encodes an unknown (and arbitrary) state $\left|\psi_{\text {in }}\right\rangle=\cos (\theta / 2)|0\rangle+e^{i \phi} \sin (\theta / 2)|1\rangle$ at site $A$ in the graph and lets the system evolve freely for a finite time $t=t_{0}$ at which the quantum state localizes at another site $B$ in the many-body system. This free quantum evolution of the entire network is precisely the quantum walk on the corresponding graph $G$ corresponding to the many-body network.

To define a quantum walk and state transfer on a graph $G=(V, E)$ of $n=|V|$ vertices, $n$-qubit states are considered that are localized at the vertices of the graph or, equivalently, the excitation space isomorphic to $\mathbb{C}^{|V|}$. A continuous-time quantum walk on a graph $G$ is the Schrödinger evolution of the graph composite state with the graph adjacency matrix $A(G)$ as the Hamiltonian [34]. If $|\zeta(0)\rangle \in \mathbb{C}^{|V|}$ is the initial quantum state, then the evolution of the quantum walk is given by

$$
|\zeta(t)\rangle=\exp [-i t A(G)]|\zeta(0)\rangle \text {. }
$$

The probability of getting the quantum state localized at the vertex $v$ at time $t$ is given by $|\langle v \mid \zeta(t)\rangle|^{2}$. Further, $G$ has a PST 
from vertex $u$ to vertex $v$ at finite time $t_{0}$ if

$$
\left|\left\langle v\left|\exp \left[-i t_{0} A(G)\right]\right| u\right\rangle\right|=1 .
$$

This is the same condition for perfect state transfer expressed in graph theoretic fashion [35] and implies Eq. (1). The graph $G$ allows a perfect state transfer from the vertex $u$ to $v$ if the $(u, v)$ th term of $\exp [-i t A(G)]$ has magnitude 1 . Similarly, Laplacian PST is when the $(u, v)$ th term of $\exp [-i t L(G)]$ has magnitude 1.

The following are some well-known examples of graphs which allow perfect state transfer over long distances $[44,45]$.

(i) The complete graph $K_{2}$ with two vertices allows perfect state transfer between its vertices in time $t_{0}=\pi / 2$ (in units of the energy inverse).

(ii) The path graph $P_{3}$ has perfect state transfer between its end vertices in time $t_{0}=\pi / \sqrt{2}$ (in units of the energy inverse).

(iii) The hypercube of any order has perfect state transfer between its antipodal points in the same time $\pi / 2$. In addition, any order of Cartesian product of $P_{3}$ has PST between its antipodal vertices in the same time $\pi / \sqrt{2}$.

These three results hold for both the $X Y$-coupling and Heisenberg interactions. We make use of these results in order to establish a process for PST between any pair of vertices in a hypercube of any dimension.

\section{MEMORY-ENHANCED PERFECT STATE TRANSFER ON HYPERCUBES}

A hypercube of dimension $n$, denoted by $\mathcal{Q}_{n}$, is a graph on $2^{n}$ vertices $(n \geqslant 0)$ which can be defined as an $n$-time Cartesian product of the complete graph on two vertices, which is an edge. Let the vertex set of the complete graph on two vertices be $\{0,1\}$. Then the vertices of $\mathcal{Q}_{n}$ are labeled as the $n$-tuples of 0 and 1 , that is, the vertex set of $\mathcal{Q}_{n}$ is $\mathcal{V}_{n}=\{0,1\}^{n}$. Two vertices $x$ and $y$ of $\mathcal{Q}_{n}$ are linked by an edge if the Hamming distance of $x, y \in\{0,1\}^{n}$ is 1 . Two vertices $x=\left(x_{1}, \ldots, x_{n}\right)$ and $y=\left(y_{1}, \ldots, y_{n}\right)$ of $Q_{n}$ are called antipodal if $x_{i} \neq y_{i}, i \in\{1, \ldots, n\}$. Given two vertices $x$ and $y$, we are interested in determining the unique induced subhypercube $\mathcal{Q}_{d}$ for some $d$ of $\mathcal{Q}_{n}(n \geqslant d)$ such that the vertices $x$ and $y$ of $\mathcal{Q}_{n}$ are antipodal in $\mathcal{Q}_{d}$. The following proposition describes the same.

Proposition 1. Let $x=\left(x_{1}, \ldots, x_{n}\right)$ and $y=\left(y_{1}, \ldots, y_{n}\right) \in$ $\mathcal{V}_{n}$, the vertex set of $\mathcal{Q}_{n}$. Suppose $d=\mid\left\{i: x_{i} \neq y_{i}, i=\right.$ $1, \ldots, n\} \mid$. Then the unique induced subhypercube $\mathcal{Q}_{d}$ of $\mathcal{Q}_{n}$ with $x$ and $y$ as antipodal vertices of $\mathcal{Q}_{d}$ is given by the set of vertices

$$
\begin{gathered}
\mathcal{V}_{d}=\left\{z=\left(z_{1}, \ldots, z_{n}\right) \in \mathcal{V}_{n}: z_{i}=x_{i} \text { if } x_{i}=y_{i}\right. \text { and } \\
\left.z_{i} \in\{0,1\} \text { otherwise, } i=1, \ldots, n\right\} .
\end{gathered}
$$

Proof. Note that if $d=n$ then $x$ and $y$ are antipodal vertices of $\mathcal{Q}_{n}$. Now let $n-d \neq 0$. Then there are indices $i_{1}, \ldots, i_{n-d}$ such that $x_{i_{j}}=y_{i_{j}}, j \in\{1, \ldots, n-d\}$. Consider the set of vertices

$$
\begin{gathered}
\mathcal{V}_{d}=\left\{z=\left(z_{1}, \ldots, z_{n}\right) \in\{0,1\}^{n}: z_{i_{j}}=x_{i_{j}}=y_{i_{j}},\right. \\
j=1, \ldots, n-d\} .
\end{gathered}
$$

Then $\left|\mathcal{V}_{d}\right|=2^{d}$. Consider the subgraph of $\mathcal{Q}_{n}$ that is induced by the vertex set $\mathcal{V}_{d}$. Thus $z, w \in \mathcal{V}_{d}$ are linked by an edge in the induced subgraph if and only if $z$ and $w$ are linked by an edge in $\mathcal{Q}_{n}$, that is, the Hamming distance between $z$ and $w$ is 1. The uniqueness of the subhypercube $\mathcal{Q}_{d}$ of $\mathcal{Q}_{n}$ follows from the fact that it is an induced subgraph of $\mathcal{Q}_{n}$. This completes the proof.

Now we propose a procedure to utilize the induced $d$ dimensional subhypercubes of an $n$-dimensional hypercube $\mathcal{Q}_{n}(n \geqslant d)$ for perfect transfer of qubits between any pair of nodes in $\mathcal{Q}_{n}$.

In the following we describe how a classical $n$-bit or $n$-qubit quantum memory address register corresponding to every vertex of $\mathcal{Q}_{n}$ can help to obtain the desired state transfer between a pair of arbitrary vertices of $\mathcal{Q}_{n}$. As discussed above, each vertex of $\mathcal{Q}_{n}$ can be labeled as an element of $\{0,1\}^{n}$. Let $x=\left(x_{1}, \ldots, x_{n}\right) \in \mathcal{V}_{n}=\{0,1\}^{n}$. Then consider a classical $n$-bit memory which stores the labeling of a vertex or quantum memory register which stores the corresponding $n$-qubit product state $|x\rangle$ for the vertex $x$. For the classical memory design and architecture, it is a standard procedure that the digital information of the classical bit 0 or 1 can be stored by distinguishing between different values of some continuous physical quantity, such as voltage or current. In addition, many organizations are possible for a memory design based on the choices of the number of cells in a memory and each cell contains a fixed number of bits (see $[52,53])$. Setting $n$ bits of data in a cell, a memory of $n 2^{n}$ bits of data is possible having $2^{n}$ memory addresses. In the present standard of architecture a large number of bits can be stored in a memory; for example, in semiconductor memory devices millions of bits can be stored and accessed [52]. Each cell is labeled with a memory address and a desired collection of addresses can be accessed by a program efficiently [54]. Hence the vertices with desired bits at the desired positions in the cells corresponding to all the vertices of the hypercube can be determined and the vertices of the subhypercube $\mathcal{Q}_{d}$ can be identified. On the other hand, observe that $\left\{|x\rangle: x \in \mathcal{V}_{n}\right\}$ is the canonical orthonormal basis of $\left(\mathbb{C}^{2}\right)^{\otimes n}$. Like a classical memory register, qubits are assumed to be placed in a quantum memory register such that each qubit in a cell can be accessed for a task such as measurement [55]. Upon measurement of the $d$ qubits in the $n$-qubit register at desired vertices (in which the measurement result matches for both $|x\rangle$ and $|y\rangle$ ), positions corresponding to every vertex can be obtained. These vertices constitute the vertex set of the desired subhypercube $\mathcal{Q}_{d}$. However, the proposal for the design and architecture of quantum memories is at the nascent stage of research (see [56,57] and references therein).

Given a pair of vertices $x$ and $y$ of $\mathcal{Q}_{n}$, finding the vertices in $\mathcal{Q}_{n}$ with the number of places where the bits of labeling of $x$ and $y$ coincide may apparently be seen as a daunting task. However, the largest semiconductor memory chips available in today's technology can hold a few gibibits 
of data, where a gibibit is equivalent to $2^{30}$ bits of data [58]. In addition, checking the bits at some fixed positions in all the memory registers can be done in parallel in $O(1)$ time. For a quantum memory register that consists of all the canonical $n$-qubit product states, each corresponding to a vertex of the hypercube $\mathcal{Q}_{n}$, the bottleneck becomes the performance of quantum measurement for each qubit at desired positions of all the $n$-qubit product states. Finally, we mention that the identification of the desired vertices of the subhypercube is independent of the switching procedure described below.

Once the list of such vertices is identified, a switching technique is proposed to create an induced subhypercube $\mathcal{Q}_{d}$ of $\mathcal{Q}_{n}$ such that $x$ and $y$ are antipodal vertices of $\mathcal{Q}_{d}$ as defined in Proposition 1. The switching technique involves tuning of the coupling strength of all the edges in $\mathcal{Q}_{n}$ that do not belong to the induced subhypercube $\mathcal{Q}_{d}$. Indeed, once the vertex set $\mathcal{V}_{d}$ is determined, all the couplings that are incident to any vertex in $\mathcal{V}_{n} \backslash \mathcal{V}_{d}$ are deactivated. We call this process a switching process as it can be interpreted as switching off some edges of $\mathcal{Q}_{n}$ for communication and once the state is transferred to a desired site, the inactive edges are again switched on for the next job over the hypercube $\mathcal{Q}_{n}$. Note that the network state for perfect state transfer is not modified due to the switching technique, but it aids to limit the communication between the desired end point vertices of the induced subhypercube $\mathcal{Q}_{d}$, which can be done by following the procedure proposed in [18]. A proposal for a possible physical implementation of the switching technique described above is explained in the next section. Below we show that perfect state transfer with unit fidelity is possible in an ideal implementation of the proposed switching procedure. A clear illustration of the switching process is represented in Fig. 1. Starting with the hypercube $\mathcal{Q}_{n=4}$, we label the vertices $v_{i} \in \mathcal{V}_{n}$. The vertices are labeled as $v_{i}=\left(v_{i_{1}}, v_{i_{2}}, v_{i_{3}}, v_{i_{4}}\right)$, with $v_{1}=(0,0,0,0), v_{2}=(0,0,0,1)$, $v_{3}=(0,0,1,0), \quad v_{4}=(0,0,1,1), \quad v_{5}=(0,1,0,0), \quad v_{6}=$ $(0,1,0,1), v_{7}=(0,1,1,0), v_{8}=(0,1,1,1)$, and $v_{2^{4}-i+1}=$ $\bar{v}_{i}=v_{i} \oplus(1,1,1,1)$, the antipodal of $v_{i}, i=1, \ldots, 8$. When switching to a hypercube, for instance, $\mathcal{Q}_{d=2}$, we have the vertex set $\left\{v_{1}, v_{2}, v_{5}, v_{6}\right\}$ in accordance with Proposition 1 , with antipodal vertex pairs $\left(v_{1}, v_{6}\right)$ and $\left(v_{2}, v_{5}\right)$. Similarly, it follows for $d=3$ and $d=1$.

Let us define the graph obtained after applying the switching techniques as $\overline{\mathcal{Q}}_{n}=\mathcal{Q}_{d} \sqcup\left\{v_{i_{1}}\right\} \sqcup \cdots \sqcup\left\{v_{i_{2^{-2 d}}}\right\}$, where $\mathcal{Q}_{d}$ is a $d$-dimensional induced subhypercube of $\mathcal{Q}_{n}^{-2^{d}}$ and $v_{i_{j}}(j=$ $1, \ldots, n-d)$ are isolated vertices of $\mathcal{Q}_{n}$. Let $A=\left[a_{i j}\right]$ be the adjacency matrix associated with $\mathcal{Q}_{n}$. Then the adjacency matrix $\bar{A}=\left[\bar{a}_{i j}\right]$ corresponding to $\overline{\mathcal{Q}}_{n}$ is given by $\bar{a}_{i j}=a_{i j}$ if $v_{i}, v_{j} \in \mathcal{V}_{d}$ and 0 otherwise.

Then we show that perfect state transfer is possible between any two antipodal vertices of the induced subhypercube $\mathcal{Q}_{d}$ of $\overline{\mathcal{Q}}_{n}$. For this we show that the PST dynamics of $\overline{\mathcal{Q}}_{n}$ is identical to that of $\mathcal{Q}_{d}$.

The graph $\mathcal{Q}_{d}$ alone in isolation will have perfect state transfer between two vertices $x$ and $y$ at time $t_{0}$. Also, let $\overline{\mathcal{Q}}_{d}$ be the fully disconnected graph with the vertex set $\mathcal{V}_{n} \backslash \mathcal{V}_{d}$. Clearly, $\overline{\mathcal{Q}}_{d}$ has no edge in itself or with $\mathcal{Q}_{d}$; hence no matrix element via adjacency matrix or Laplacian exists that can exchange an excitation involving these vertices. The PST in isolated $\mathcal{Q}_{d}$ indicates $\left|\left\langle y\left|\exp \left[-i t_{0} A\left(\mathcal{Q}_{d}\right)\right]\right| x\right\rangle\right|=1$, where $|x\rangle$

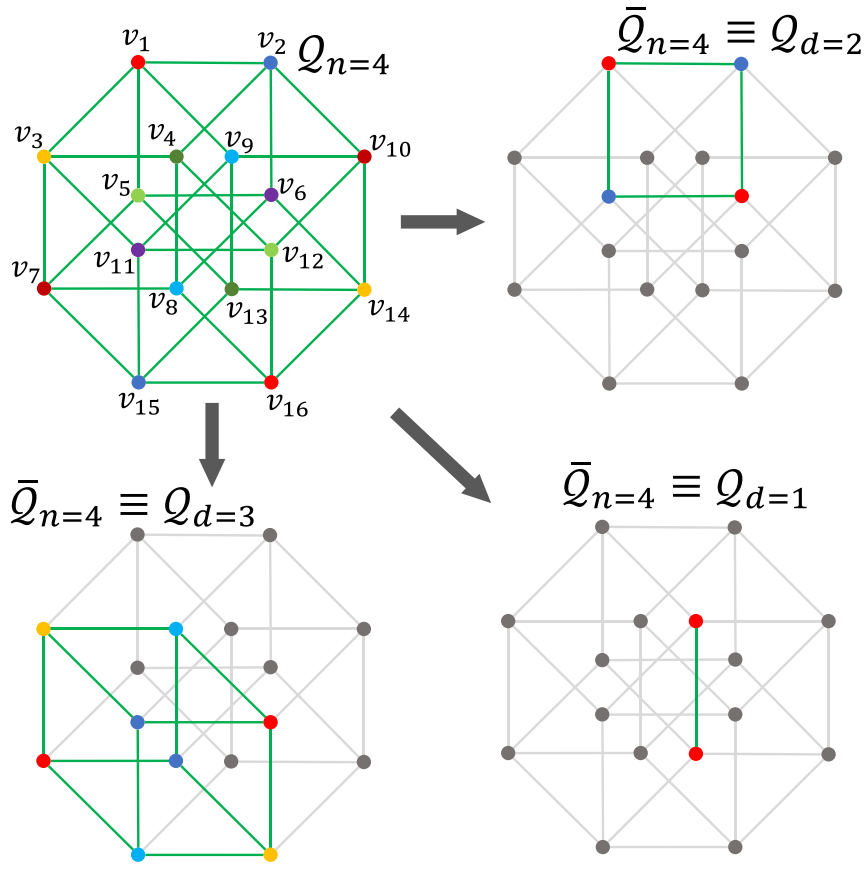

FIG. 1. Visual representation of the switching process. Green represents switched-on edges and gray indicates switched-off edges. Isolated vertices are in dark gray. Any hypercube $\mathcal{Q}_{n}$ (here $n=4$ ) under switching can realize various embedded subhypercubes $\left(\overline{\mathcal{Q}}_{n}\right.$ is effectively identical to $\mathcal{Q}_{d}$ for PST dynamics). Antipodal vertices of the active hypercubes $\mathcal{Q}_{d}$ are denoted by the same the color.

and $|y\rangle$ are the canonical basis vectors of $\mathbb{C}^{2^{d}}$ corresponding to the vertices $x$ and $y$, respectively.

Since $\overline{\mathcal{Q}}_{d}$ contains $2^{n}-2^{d}$ vertices, we know that the graph $\mathcal{Q}_{d} \sqcup \overline{\mathcal{Q}}_{d} \equiv \overline{\mathcal{Q}}_{n}$ has $2^{n}$ vertices. Corresponding to the vertices $x$ and $y$, we define state vectors in $\mathbb{C}^{2^{n}}$ as $\left|x^{\prime}\right\rangle=\left[\begin{array}{c}|x\rangle \\ {\left[2^{n}-2^{d} \mid \times 1\right.}\end{array}\right]$ and $\left.\left|y^{\prime}\right\rangle={ }_{\mathbf{0}_{\left|2^{n}-^{d}\right| \times 1}}{ }^{\mid l y}\right]$, respectively. Now the adjacency matrix of $\overline{\mathcal{Q}}_{n}$ takes the block form

$$
A\left(\overline{\mathcal{Q}}_{n}\right)=\left[\begin{array}{cc}
A\left(\mathcal{Q}_{d}\right) & \mathbf{0}_{\left|2^{d}\right| \times\left|2^{n}-2^{d}\right|} \\
\mathbf{0}_{\left|2^{n}-2^{d}\right| \times\left|2^{d}\right|} & A\left(\overline{\mathcal{Q}}_{d}\right)
\end{array}\right] .
$$

However, the adjacency matrix $A\left(\overline{\mathcal{Q}}_{d}\right)$ is a zero matrix because there is no interaction among the isolated vertices in $\overline{\mathcal{Q}}_{d}$. Then the evolution matrix is

$$
\exp \left[-i t_{0} A\left(\overline{\mathcal{Q}}_{n}\right)\right]=\left[\begin{array}{cc}
\exp \left[-i t_{0} A\left(\mathcal{Q}_{d}\right)\right] & \mathbf{0}_{\left|2^{d}\right| \times\left|2^{n}-2^{d}\right|} \\
\mathbf{0}_{\left|2^{n}-2^{d}\right| \times\left|2^{d}\right|} & I
\end{array}\right] .
$$

Now the condition for PST between $x$ and $y$ in $\overline{\mathcal{Q}}_{n}$ is

$$
\begin{aligned}
& \left|\left\langle y^{\prime}\left|\exp \left[-i t_{0} A\left(\overline{\mathcal{Q}}_{n}\right)\right]\right| x^{\prime}\right\rangle\right|
\end{aligned}
$$

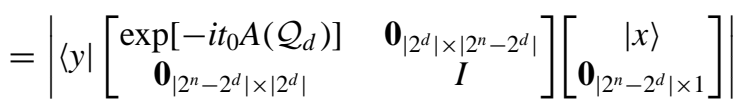

$$
\begin{aligned}
& =\mid\left[\langle y| \mathbf{0}_{1 \times\left|2^{n}-2^{d}\right|}\right]\left[\begin{array}{c}
\exp \left[-i t_{0} A\left(\mathcal{Q}_{d}\right)\right]|x\rangle \\
\mathbf{0}_{\left|2^{n}-2^{d}\right| \times 1}
\end{array}\right] \mid \\
& =\left|\left\langle y\left|\exp \left[-i t_{0} A\left(\mathcal{Q}_{d}\right)\right]\right| x\right\rangle\right|=1 \text {. }
\end{aligned}
$$

Therefore, the graph $\overline{\mathcal{Q}}_{n}$ has identical perfect state transfer dynamics like that of the graph $\mathcal{Q}_{d}$. This guarantees PST in the 
(a)

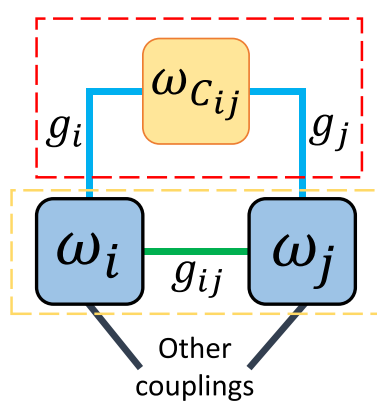

_ - Part of the implementation

_ - Part of our architecture

FIG. 2. (a) Couplings involved between a pair of interacting qubits, forming an edge in the hypercube $\mathcal{Q}_{n}$. (b) Network of four qubits forming $\mathcal{Q}_{2}$. Each ancillary coupler is associated with every edge which is controlled in the experiment.

induced subhypercube $\mathcal{Q}_{d}$ under switching. A similar argument can be given considering the Laplacian matrix associated with the hypercube in place of the adjacency matrix which corresponds to the Heisenberg model.

\section{PROPOSAL FOR IMPLEMENTATION OF SWITCHING OPERATION WITH SUPERCONDUCTING QUBITS}

In this section we describe the physical realization for the hypercube switching PST under the $X Y$ coupling. The same architecture holds true for the Cartesian products of the $P_{3}$ graph, the path graph on three vertices. We propose the implementation of our PST scheme as pretty good state transfer with a fidelity of $\mathcal{F}=1-O\left((g / \Delta)^{3}\right)$, where $g$ is related to the strength of the qubit-qubit coupling and $\Delta$ is the detuning of qubits. Our task is to show support for switching of the hypercube $\mathcal{Q}_{n}$ to $\overline{\mathcal{Q}}_{n}$ as desired for any pair of chosen vertices of $\mathcal{Q}_{n}$ for the task of PST. The following are key physical requirements of our architecture: (a) $n$ nearest-neighbor interactions to realize any general $\mathcal{Q}_{n}$ hypercube, (b) three-dimensional integration $[59,60]$ (since distant qubits are connected, implementation is not possible in planar integration, $\mathcal{Q}_{4}$ onward), (c) tunable (switchable) edges as couplings for each pair of nodes (qubits), and (d) high-fidelity control over the processor [61].

Most of these requirements are hard to realize with the conventional spin lattice where switching and tuning of edge coupling become highly challenging. Moreover, such coupling is a function of the distance between two nodes, which is not changeable in practice. Instead, to implement our network we consider a different adjustable coupler that involves ancillary qubits acting as (tunable) couplers [39,62,63] (see Fig. 2). These additional couplers linked to architectural qubits often add complexity and create an alternative means for decoherence [64]. However, superconducting qubits have already sufficiently long decoherence times $[39,63]$ and high-fidelity gates have been realized using tunable couplers [65].

We consider a general system that consists of $2^{n}$ qubits for $\mathcal{Q}_{n}$ with exchange coupling between nearest qubits (which have an edge between them). In addition, as anticipated, a switchable coupling also requires an extra qubit between them. The total number of these ancillary couplers is equal to the number of edges in the network $\left(n 2^{n-1}\right.$ for $\left.\mathcal{Q}_{n}\right)$. We use the denotation $i$ and $j$ for qubits and $C_{i j}$ for the coupler that connects to these qubits. Both the qubits $i$ and $j$ (with respective Zeeman splittings $\omega_{i}$ and $\omega_{j}$ ) couple to the auxiliary one $\left(\omega_{C_{i j}}\right)$ with a strength $g_{i}\left(i=1,2, \ldots, 2^{n}\right)$. In addition, they are mutually coupled with transverse coupling strength $g_{i j}$. The total Hamiltonian including both physical and ancillary qubits is given by

$$
H_{\mathcal{Q}_{n}}=\sum_{\langle i, j\rangle}\left(H_{i}+H_{j}+H_{c_{i j}}+H_{i c_{i j}}+H_{j c_{i j}}+H_{i j}\right),
$$

where $\langle i, j\rangle:=(i, j) \in E\left(\mathcal{Q}_{n}\right)$ defines the pair of adjacent vertices in $\mathcal{Q}_{n}$. Explicitly,

$$
\begin{aligned}
\frac{H_{\mathcal{Q}_{n}}}{\hbar}= & \frac{1}{2} \sum_{i=1}^{2^{n}} \omega_{i} \sigma_{i}^{z}+\frac{1}{2} \sum_{\langle i, j\rangle} \omega_{C_{i j}} \sigma_{C_{i j}}^{z}+\sum_{\langle i, j\rangle} g_{i} \sigma_{i}^{x} \sigma_{C_{i j}}^{x} \\
& +\sum_{\langle i, j\rangle} g_{i j} \sigma_{i}^{x} \sigma_{j}^{x} .
\end{aligned}
$$

If we consider capacitive qubit-qubit coupling, which is advantageous in terms of decoherence times, the coupling strengths can be estimated [63] as

$$
g_{j}=\frac{1}{2} \frac{C_{j c_{i j}}}{\sqrt{C_{j} C_{c_{i j}}}} \sqrt{\omega_{j} \omega_{C_{i j}}}
$$

and

$$
g_{i j}=\frac{1}{2}\left(1+\eta_{i j}\right) \frac{C_{i j}}{\sqrt{C_{i} C_{j}}} \sqrt{\omega_{i} \omega_{j}},
$$

where $\eta_{i j}=C_{i c_{i j}} C_{j c_{i j}} / C_{i j} C_{c_{i j}}$. Here $C_{\lambda}$ is the transmon qubit capacitance, $C_{i j}$ is the direct qubit-qubit coupling capacitance, $C_{i c_{i j}}$ is the qubit-coupler coupling capacitance, and $C_{c_{i j}}$ is the coupler capacitance. In the following we consider the socalled dispersive regime in which the qubits are well detuned, i.e., $g_{i} \ll\left|\Delta_{i}\right| \forall i$, with $\Delta_{i}=\omega_{i}-\omega_{C_{i j}}<0$ the qubit-ancilla detuning. Therefore, we can use a perturbation theory in $g_{i} / \Delta_{i}$. In order to do so, it is convenient to use the SchriefferWolff (SW) unitary transformation $U_{\mathrm{SW}}=e^{\eta}$ [66]. In our case,

$$
\begin{array}{r}
U_{\mathrm{SW}}=\exp \left[\sum _ { \langle i , j \rangle } \left(\frac{g_{i}}{\Delta_{i}}\left(\sigma_{i}^{+} \sigma_{C_{i j}}^{-}-\sigma_{i}^{-} \sigma_{C_{i j}}^{+}\right)\right.\right. \\
\left.\left.+\frac{g_{i}}{\Sigma_{i}}\left(\sigma_{i}^{+} \sigma_{C_{i j}}^{+}-\sigma_{i}^{-} \sigma_{C_{i j}}^{-}\right)\right)\right],
\end{array}
$$

where the second term takes care of the counterrotating terms and $\Sigma_{i}=\omega_{i}+\omega_{C_{i j}}$. The transformation $U_{\mathrm{SW}} H U_{\mathrm{SW}}^{\dagger}$ is done up to second order in $g_{i} / \Delta_{i}$. In addition, it is assumed that the ancillary qubits always remain in their ground state, which is consistent with the condition $\Delta_{i}=\omega_{i}-\omega_{C_{i j}}<0$. After some algebra we end up with the effective qubit-qubit interaction Hamiltonian (see Appendix A)

$$
\frac{\tilde{V}}{\hbar}=\sum_{\langle i, j\rangle} \tilde{J}_{i j}\left(\sigma_{i}^{+} \sigma_{j}^{-}+\sigma_{i}^{-} \sigma_{j}^{+}\right),
$$




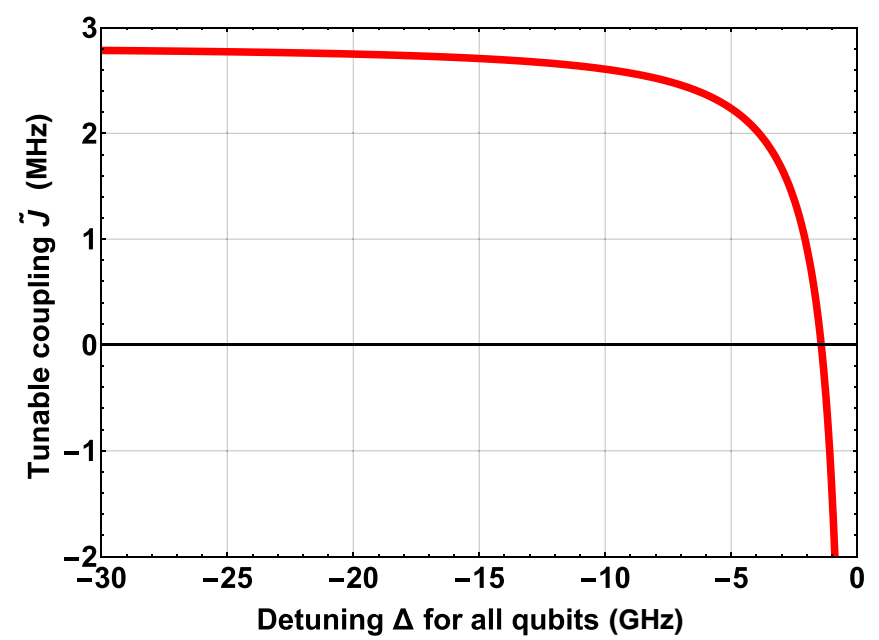

FIG. 3. Variation of the dynamic tunable coupling $\tilde{J}$ with respect to the detuning $\Delta$ for each qubit. There exists a cutoff value, in this case $\Delta=-1.426 \mathrm{GHz}$, corresponding to $\omega_{C_{i j}}^{\text {off }}=5.426 \mathrm{GHz}$. For all configurations, such a cutoff value can always be obtained.

where the effective tunable coupling between any two qubits is given by

$$
\tilde{J}_{i j} \approx \frac{g_{i} g_{j}}{2}\left(\frac{1}{\Delta_{i}}+\frac{1}{\Delta_{j}}-\frac{1}{\Sigma_{i}}-\frac{1}{\Sigma_{j}}\right)+g_{i j}
$$

Similar effective coupling Hamiltonians based on cavity and circuit QED have been proposed in [67] (scalability has been addressed with experimental concerns using molecular architecture for qubits in superconducting resonators) and [8]. It is clear that the above produces the identical coupling Hamiltonian in Eq. (2) which is responsible for qubit-qubit hopping; however, now the hopping term is tunable by setting the desired couplings $\left(2 J_{i j} \longrightarrow \tilde{J}_{i j}\right)$ and detunings. In Fig. 3 we show how $\tilde{J}_{i j}$ can be changed to negative when the ancilla coupler frequency is reduced or changed to positive when this frequency is escalated. Therefore, we have some $\omega_{C_{i j}}^{\mathrm{off}}$ such that $\tilde{J}_{i j}\left(\omega_{C_{i j}}^{\mathrm{off}}\right)=0$ within the bandwidth of each coupler. It has been shown [63] that this cutoff frequency can be found even in a weak dispersive regime with $g_{j}<\left|\Delta_{j}\right|$. Thus, we obtain the switchable edges with $\omega_{C_{i j}}$ as the parameter. We can simply tune each frequency $\omega_{C_{i j}}$ for each edge $E(i, j)$ to switch it on or off whenever our protocol requires switching to the graph $\overline{\mathcal{Q}}_{n}$ from $\mathcal{Q}_{n}$ and this is essentially a classical operation in experiment. The couplers remain in their ground state throughout the quantum evolution as the effective interaction is only for one quantum exchange between the two qubits which are part of the network for $\mathcal{Q}_{n}$. For the case when all qubits are capacitively coupled and $\omega_{i}=$ $\omega_{j}=\omega$, using Eqs. (13) and (14), the effective coupling is simplified to

$$
\tilde{J}_{i j}=\frac{1}{2}\left[\frac{\omega^{2}}{\Delta \Sigma} \eta_{i j}+1\right] \frac{C_{i j}}{\sqrt{C_{i} C_{j}}} \omega .
$$

In conclusion, to switch on the qubit-qubit coupling as an edge in the network graph, one sets the respective edge coupler's detuning to a reasonable (within the bandwidth) value via $\omega_{C_{i j}}^{\mathrm{on}}$, yielding a limited $\tilde{J}\left(\omega_{C_{i j}}^{\mathrm{on}}\right)$. Then a PST can be performed by modulating only the couplers' frequency to $\tilde{J}\left(\omega_{C_{i j}}^{\mathrm{on}}\right)$ for all the edges $E\left(\mathcal{Q}_{d}\right)$. Therefore,

$$
\tilde{V}_{\mathcal{Q}_{n}} \stackrel{\text { switch off } E\left(\mathcal{Q}_{n}-\mathcal{Q}_{d}\right)}{\longrightarrow} \tilde{V}_{\overline{\mathcal{Q}}_{n} \equiv \mathcal{Q}_{d}}
$$

and the perfect state transfer time $\left(t_{0}\right)$ is related to the dynamical coupling as $t_{0}=\pi / 2 \tilde{J}^{\mathrm{on}}$.

Figure 3 shows the variation (18) of the dynamic tunable coupling $\tilde{J}$ with respect to the control parameter $\omega_{C_{i j}}$ through $\Delta$. The reasonable experimental values for the parameters used are [63] $C_{i}=70 \mathrm{fF}, C_{j}=72 \mathrm{fF}, C_{c_{i j}}=200 \mathrm{fF}, C_{i c_{i j}}=4$ fF, $C_{j c_{i j}}=4.2 \mathrm{fF}$, and $C_{i j}=0.1 \mathrm{fF}$. All $\omega_{i}=\omega_{j}=\omega=4 \mathrm{GHz}$ (because all qubits are identical). The fabrication defects and imperfection are accounted for in the different values for the capacitance. We have to ensure in the experiment that all detunings are set to the same value to realize a uniformly coupled qubit hypercube $\mathcal{Q}_{d}$. If all detunings are not equal this will actually realize a weighted edge hypercube and introduce an error in the fidelity (see Appendix B). In one way, it can be first compensated by different values of the capacitive couplings involved. For the error that still remains, we can calculate the bound on the fidelity. For the case of the example in Fig. 1 with $\mathcal{Q}_{n=4}$, that the maximum deviation in $\tilde{J}_{i j}$ for each edge is $\pm 0.5 \%$, we have $\mathcal{F}>97.43 \%$ [using Eq. (B8)].

\section{CONCLUSION}

We have proposed a switching procedure on a memoryenhanced hypercube such that an induced subhypercube can be determined with a desired pair of antipodal vertices of the hypercube. Consequently, we showed that the constructed subhypercube enables one to provide support for perfect state transfer between any pair of distinct vertices of the hypercube. It is clear that the proposed method can be scaled up to any higher-dimensional hypercube except that the access and storage of the labeling of vertices of the hypercube can be done efficiently. A framework of superconducting qubits with tunable couplings was defined for physical implementation of the switching procedure under the $X Y$ coupling. It was shown that perfect state transfer between any pair of vertices in a hypercube is possible utilizing the proposed switching procedure. It will be interesting to extend this method for graphs that have structural support of construction of subhypercubes as subgraphs of the graph on any number vertices. Also, it will be equally interesting to propose a physical implementation of our protocol for the case of the Heisenberg interaction where the $z-z$ qubit couplings are required to be tunable. These questions remain to be addressed.

\section{APPENDIX A: EFFECTIVE HAMILTONIAN}

In this Appendix we derive the effective Hamiltonian (16) with the aim of decoupling the ancillary couplers from the computational qubits. Starting with the non-rotatingwave-approximation Hamiltonian (12) and employing the Schrieffer-Wolff transformation (15), we obtain the most 
general Hamiltonian up to second order in $g_{i} / \Delta_{i}$ as

$$
\begin{aligned}
\frac{\tilde{H}_{\mathcal{Q}_{n}}}{\hbar}= & \frac{1}{2} \sum_{i=1}^{2^{n}} \omega_{i} \sigma_{i}^{z}+\frac{1}{2} \sum_{\langle i, j\rangle} \omega_{C_{i j}} \sigma_{C_{i j}}^{z}+\sum_{\langle i, j\rangle} g_{i j} \sigma_{i}^{x} \sigma_{j}^{x}+\sum_{\langle i, j\rangle}\left[-\frac{g_{i}^{2}}{2}\left(\frac{1}{\Delta_{i}}-\frac{1}{\Sigma_{i}}\right)\left(\sigma_{i}^{+} \sigma_{j}^{+}+\sigma_{i}^{-} \sigma_{j}^{-}\right) \sigma_{C_{i j}}^{z}\right. \\
& +\frac{g_{i}^{2}}{2}\left(\frac{1}{\Delta_{i}}+\frac{1}{\Sigma_{i}}\right) \sigma_{i}^{z} \sigma_{C_{i j}}^{+} \sigma_{C_{i j}}^{+}-\frac{g_{i}^{2}}{2}\left(\frac{1}{\Delta_{i}}-\frac{1}{\Sigma_{i}}\right) \sigma_{i}^{z} \sigma_{C_{i j}}^{-} \sigma_{C_{i j}}^{-}+\frac{g_{i}^{2}}{\Delta_{i}}\left(\sigma_{i}^{z} \sigma_{C_{i j}}^{-} \sigma_{C_{i j}}^{+}+\sigma_{i}^{-} \sigma_{i}^{+}\right) \\
& \left.+\frac{g_{i}^{2}}{\Sigma_{i}}\left(\sigma_{i}^{z} \sigma_{C_{i j}}^{-} \sigma_{C_{i j}}^{+}-\sigma_{i}^{+} \sigma_{i}^{-} \sigma_{C_{i j}}^{z}\right)\right]+\sum_{\langle i, j\rangle}\left[\frac{g_{i}^{2}}{2}\left(\frac{1}{\Delta_{i}}-\frac{1}{\Sigma_{i}}\right) \sigma_{i}^{z}\left(\sigma_{C_{i j}}^{-} \sigma_{C_{i k}}^{+}-\sigma_{C_{i j}}^{+} \sigma_{C_{i k}}^{-}+\sigma_{C_{i j}}^{-} \sigma_{C_{i k}}^{-}-\sigma_{C_{i j}}^{+} \sigma_{C_{i k}}^{+}\right)\right] \\
& -\sum_{\langle i, j\rangle} \frac{g_{i} g_{j}}{2}\left(\frac{1}{\Delta_{i}}+\frac{1}{\Delta_{j}}-\frac{1}{\Sigma_{i}}-\frac{1}{\Sigma_{j}}\right)\left(\sigma_{i}^{+} \sigma_{j}^{+}+\sigma_{i}^{+} \sigma_{j}^{-}+\sigma_{i}^{-} \sigma_{j}^{+}+\sigma_{i}^{-} \sigma_{j}^{-}\right) \sigma_{C_{i j}}^{z} .
\end{aligned}
$$

We now drop the terms which involve double excitation of either the qubits or the couplers and impose the strict condition that all couplers remain in their ground state. This completely decouples the coupler Hamiltonian, which can be completely dropped. Further, all the constant energy shift terms can be neglected. This results in the network Hamiltonian with renormalized frequencies

$$
\frac{\tilde{H}_{\mathcal{Q}_{n}}}{\hbar}=\frac{1}{2} \sum_{i=1}^{2^{n}} \tilde{\omega}_{i} \sigma_{i}^{z}+\underbrace{\sum_{\langle i, j\rangle} \tilde{J}_{i j}\left(\sigma_{i}^{+} \sigma_{j}^{-}+\sigma_{i}^{-} \sigma_{j}^{+}\right)}_{\tilde{V}},
$$

where $\tilde{V}$ is the effective qubit-qubit interaction,

$$
\tilde{\omega}_{i} \approx \omega_{i}+g_{i}^{2}\left(\frac{1}{\Delta_{i}}+\frac{1}{\Sigma_{i}}\right)
$$

is the corrected frequency due to the Lamb-shift frequency revealed by the SW transformation, and

$$
\tilde{J}_{i j} \approx \frac{g_{i} g_{j}}{2}\left(\frac{1}{\Delta_{i}}+\frac{1}{\Delta_{j}}-\frac{1}{\Sigma_{i}}-\frac{1}{\Sigma_{j}}\right)+g_{i j}
$$

is the effective and dynamic qubit-qubit coupling of the network qubits.

\section{APPENDIX B: ERROR CALCULATION FOR NONIDEAL SWITCHING}

Starting from the hypercube $\mathcal{Q}_{n}$ and switching to a graph $\overline{\mathcal{Q}}_{n}$ for a desired $\mathcal{Q}_{d}$, the switching may not be ideal, meaning that the detuning parameters are not exact. This means that there can be edge strengths which are not exactly zero but are coupled with some finite effective strength $\tilde{J}_{i j} \neq 0$ between the qubits $i$ and $j$ that are supposed to be decoupled. Also, there can be edges which are not all identically weighted as $\tilde{J}^{\text {on }}$. Therefore, the effective network graph adjacency matrix is weighted $\left[A^{\prime}\left(\overline{\mathcal{Q}}_{n}\right)\right]$. It is important to calculate the error bounds due to these experimental errors over the fidelity of PST. Here we look at such error independently. Equation (8) then takes the form

$$
A^{\prime}\left(\overline{\mathcal{Q}}_{n}\right)=\left[\begin{array}{cc}
A\left(\mathcal{Q}_{d}\right)+E\left(\mathcal{Q}_{d}\right) & E \\
E^{T} & E\left(\overline{\mathcal{Q}}_{d}\right)
\end{array}\right]
$$

$$
=\left[\begin{array}{cc}
A\left(\mathcal{Q}_{d}\right) & 0 \\
0 & 0
\end{array}\right]+\left[\begin{array}{cc}
E\left(\mathcal{Q}_{d}\right) & E \\
E^{T} & E\left(\overline{\mathcal{Q}}_{d}\right)
\end{array}\right]=\mathcal{U}+\mathcal{E}
$$

where the $\mathcal{E}=\left[e_{i j}\right]$ (with $\left|e_{i j}\right| \ll\left|a_{i j}\right|$ ) is a matrix with small norm that captures the effect of unwanted edges. Then from [68] we have

$$
\exp [-i t(\mathcal{U}+\mathcal{E})]=\exp (-i t \mathcal{U})+K(-i t, \mathcal{E}),
$$

where

$$
\begin{aligned}
K(-i t, \mathcal{E}) & =\int_{0}^{t} \exp [-(i t+s) \mathcal{U}] \mathcal{E} \exp [s(\mathcal{U}+\mathcal{E})] d s \\
& =\sum_{m=1}^{\infty} K_{m}(-i t, \mathcal{E})
\end{aligned}
$$

and

$$
\begin{aligned}
K_{m}(-i t, \mathcal{E}) & =\int_{0}^{t} \exp [-(i t+s) \mathcal{U}] G_{m}(s, \mathcal{E}) d s, \\
G_{m}(s, \mathcal{E}) & =\sum_{r=m-1}^{\infty} \frac{s^{r}}{r !} \sum_{i_{1}+i_{2}+\cdots+i_{m}=r-m+1} \Pi_{k=1}^{m}(\mathcal{E U})^{i_{k}} .
\end{aligned}
$$

Indeed,

$$
\left\|K_{m}(-i t, \mathcal{E})\right\|_{2}=O\left(\|\mathcal{E}\|_{2}^{m}\right) \leqslant O\left(\|\mathcal{E}\|_{F}^{m}\right),
$$

where $\|M\|_{2}=\sum_{x \neq 0} \frac{\|M x\|_{2}}{\|x\|_{2}}$ and $\|M\|_{F}=\left(\sum_{i, j=1}^{n}\left|m_{i j}\right|^{2}\right)^{1 / 2}$ for any matrix $M=\left[m_{i j}\right]$ of order $n \times n$. Therefore,

$$
\|K(-i t, \mathcal{E})\|_{2} \leqslant \sum_{m=1}^{\infty} O\left(\|\mathcal{E}\|_{F}^{m}\right) .
$$

Then modulus inequality reveals that

$$
\begin{gathered}
\left|\left\langle y^{\prime}\left|e^{-i t_{0} \mathcal{U}}\right| x^{\prime}\right\rangle+\left\langle y^{\prime}\left|K\left(-i t_{0}, \mathcal{E}\right)\right| x^{\prime}\right\rangle\right| \\
\geqslant 1-\left|\left\langle y^{\prime}\left|K\left(-i t_{0}, \mathcal{E}\right)\right| x^{\prime}\right\rangle\right| .
\end{gathered}
$$

Using Eq. (B6) equipped with the Cauchy-Schwarz inequality gives the bound on the effective fidelity as

$$
\mathcal{F} \geqslant 1-\sum_{m=1}^{\infty} O\left(\|\mathcal{E}\|_{F}^{m}\right) .
$$


Therefore, all the inaccurate edge strengths give a compromise on the fidelity which is captured by this inequality, by simply knowing the estimate for the strength of various effective coupling deviations in $\overline{\mathcal{Q}}_{n}$.
[1] C. H. Bennett and D. P. DiVincenzo, Quantum information and computation, Nature (London) 404, 247 (2000).

[2] A. Blais, A. L. Grimsmo, S. M. Girvin, and A. Wallraff, Circuit quantum electrodynamics, arXiv:2005.12667.

[3] A. F. Kockum and F. Nori, Quantum Bits with Josephson Junctions (Springer International, Cham, 2019), pp. 703-741.

[4] J. Q. You and F. Nori, Quantum information processing with superconducting qubits in a microwave field, Phys. Rev. B 68, 064509 (2003).

[5] J. Q. You and F. Nori, Atomic physics and quantum optics using superconducting circuits, Nature (London) 474, 589 (2011).

[6] I. Buluta, S. Ashhab, and F. Nori, Natural and artificial atoms for quantum computation, Rep. Prog. Phys. 74, 104401 (2011).

[7] X. Gu, A. F. Kockum, A. Miranowicz, Y.-x. Liu, and F. Nori, Microwave photonics with superconducting quantum circuits, Phys. Rep. 718-719, 1 (2017).

[8] A. Blais, R.-S. Huang, A. Wallraff, S. M. Girvin, and R. J. Schoelkopf, Cavity quantum electrodynamics for superconducting electrical circuits: An architecture for quantum computation, Phys. Rev. A 69, 062320 (2004).

[9] J. W. Britton, B. C. Sawyer, A. C. Keith, C.-C. J. Wang, J. K. Freericks, H. Uys, M. J. Biercuk, and J. J. Bollinger, Engineered two-dimensional Ising interactions in a trapped-ion quantum simulator with hundreds of spins, Nature (London) 484, 489 (2012).

[10] C. D. Bruzewicz, J. Chiaverini, R. McConnell, and J. M. Sage, Trapped-ion quantum computing: Progress and challenges, Appl. Phys. Rev. 6, 021314 (2019).

[11] R. Barends, J. Kelly, A. Megrant, A. Veitia, D. Sank, E. Jeffrey, T. C. White, J. Mutus, A. G. Fowler, B. Campbell et al., Superconducting quantum circuits at the surface code threshold for fault tolerance, Nature (London) 508, 500 (2014).

[12] M. W. Johnson, M. H. S. Amin, S. Gildert, T. Lanting, F. Hamze, N. Dickson, R. Harris, A. J. Berkley, J. Johansson, P. Bunyk et al., Quantum annealing with manufactured spins, Nature (London) 473, 194 (2011).

[13] L.-A. Wu, A. Miranowicz, X. Wang, Y.-x. Liu, and F. Nori, Perfect function transfer and interference effects in interacting boson lattices, Phys. Rev. A 80, 012332 (2009).

[14] M. Markiewicz and M. Wieśniak, Perfect state transfer without state initialization and remote collaboration, Phys. Rev. A 79, 054304 (2009).

[15] D. Burgarth, V. Giovannetti, and S. Bose, Efficient and perfect state transfer in quantum chains, J. Phys. A: Math. Gen. 38, 6793 (2005).

[16] H. Bernien, S. Schwartz, A. Keesling, H. Levine, A. Omran, H. Pichler, S. Choi, A. S. Zibrov, M. Endres, M. Greiner, V. Vuletić, and M. D. Lukin, Probing many-body dynamics on a 51-atom quantum simulator, Nature (London) 551, 579 (2017).

[17] C. Song, K. Xu, W. Liu, C.-p. Yang, S.-B. Zheng, H. Deng, Q. Xie, K. Huang, Q. Guo, L. Zhang, P. Zhang, D. Xu, D. Zheng, X. Zhu, H. Wang, Y.-A. Chen, C.-Y. Lu, S. Han, and J.-W. Pan, 10-Qubit Entanglement and Parallel Logic Operations with a Superconducting Circuit, Phys. Rev. Lett. 119, 180511 (2017).
[18] J. Kelly, R. Barends, A. G. Fowler, A. Megrant, E. Jeffrey, T. C. White, D. Sank, J. Y. Mutus, B. Campbell, Y. Chen et al., State preservation by repetitive error detection in a superconducting quantum circuit, Nature (London) 519, 66 (2015).

[19] D. P. DiVincenzo, The physical implementation of quantum computation, Fortschr. Phys. 48, 771 (2000).

[20] S. Bose, Quantum Communication through an Unmodulated Spin Chain, Phys. Rev. Lett. 91, 207901 (2003).

[21] T. J. Osborne, Statics and dynamics of quantum $X Y$ and Heisenberg systems on graphs, Phys. Rev. B 74, 094411 (2006).

[22] A. Kay, Basics of perfect communication through quantum networks, Phys. Rev. A 84, 022337 (2011).

[23] A. Ajoy and P. Cappellaro, Mixed-state quantum transport in correlated spin networks, Phys. Rev. A 85, 042305 (2012).

[24] A. Kay, Perfect, efficient, state transfer and its applications as a constructive tool, Int. J. Quantum Inf. 08, 641 (2010).

[25] V. Subrahmanyam, Entanglement dynamics and quantum-state transport in spin chains, Phys. Rev. A 69, 034304 (2004).

[26] A. Wojcik, T. Luczak, P. Kurzynski, A. Grudka, T. Gdala, and M. Bednarska, Multiuser quantum communication networks, Phys. Rev. A 75, 022330 (2007).

[27] G. Coutinho and H. Liu, No Laplacian perfect state transfer in trees, SIAM J. Discrete Math. 29, 2179 (2015).

[28] E. Ackelsberg, Z. Brehm, A. Chan, J. Mundinger, and C. Tamon, Laplacian state transfer in coronas, Linear Algebra Appl. 506, 154 (2016).

[29] R. Sousa and Y. Omar, Pretty good state transfer of entangled states through quantum spin chains, New J. Phys. 16, 123003 (2014).

[30] L. Banchi, G. Coutinho, C. Godsil, and S. Severini, Pretty good state transfer in qubit chains-The Heisenberg Hamiltonian, J. Math. Phys. 58, 032202 (2017).

[31] A. Kay, Perfect and pretty good state transfer for field-free Heisenberg chains, arXiv:1906.06223.

[32] W. J. Chetcuti, C. Sanavio, S. Lorenzo, and T. J. G. Apollaro, Perturbative many-body transfer, New J. Phys. 22, 033030 (2020)

[33] L. Banchi, A. Bayat, P. Verrucchi, and S. Bose, Nonperturbative Entangling Gates between Distant Qubits Using Uniform Cold Atom Chains, Phys. Rev. Lett. 106, 140501 (2011).

[34] V. M. Kendon and C. Tamon, Perfect state transfer in quantum walks on graphs, J. Comput. Theor. Nanosci. 8, 422 (2011).

[35] J. Brown, C. Godsil, D. Mallory, A. Raz, and C. Tamon, Perfect state transfer on signed graphs, Quantum Inf. Comput. 13, 511 (2013).

[36] C. Godsil and S. Lato, Perfect state transfer on oriented graphs, arXiv:2002.04666.

[37] M. Asoudeh and V. Karimipour, Perfect state transfer on spin-1 chains, Quantum Inf. Process. 13, 601 (2014).

[38] M. A. Jafarizadeh, R. Sufiani, M. Azimi et al., Perfect state transfer over interacting boson networks associated with group schemes, Quantum Inf. Process. 11, 171 (2012).

[39] F. Arute, K. Arya, R. Babbush, D. Bacon, J. C. Bardin, R. Barends, R. Biswas, S. Boixo, Fernando G. S. L. Brandao et al., 
Quantum supremacy using a programmable superconducting processor, Nature (London) 574, 505 (2019).

[40] J. I. Cirac and P. Zoller, Quantum Computations with Cold Trapped Ions, Phys. Rev. Lett. 74, 4091 (1995).

[41] A. Blais, J. Gambetta, A. Wallraff, D. I. Schuster, S. M. Girvin, M. H. Devoret, and R. J. Schoelkopf, Quantum-information processing with circuit quantum electrodynamics, Phys. Rev. A 75, 032329 (2007).

[42] Y.-x. Liu, C. P. Sun, and F. Nori, Scalable superconducting qubit circuits using dressed states, Phys. Rev. A 74, 052321 (2006).

[43] X. Li, Y. Ma, J. Han, T. Chen, Y. Xu, W. Cai, H. Wang, Y. P. Song, Z.-Y. Xue, Z.-q. Yin, and L. Sun, Perfect Quantum State Transfer in a Superconducting Qubit Chain with Parametrically Tunable Couplings, Phys. Rev. Appl. 10, 054009 (2018).

[44] M. Christandl, N. Datta, A. Ekert, and A. J. Landahl, Perfect State Transfer in Quantum Spin Networks, Phys. Rev. Lett. 92, 187902 (2004)

[45] M. Christandl, N. Datta, T. C. Dorlas, A. Ekert, A. Kay, and A. J. Landahl, Perfect transfer of arbitrary states in quantum spin networks, Phys. Rev. A 71, 032312 (2005).

[46] S. Bose, A. Casaccino, S. Mancini, and S. Severini, Communication in XYZ all-to-all quantum networks with a missing link, Int. J. Quantum Inf. 07, 713 (2009).

[47] C. Facer, J. Twamley, and J. Cresser, Quantum Cayley networks of the hypercube, Phys. Rev. A 77, 012334 (2008).

[48] S. Paganelli, S. Lorenzo, T. J. G. Apollaro, F. Plastina, and G. L. Giorgi, Routing quantum information in spin chains, Phys. Rev. A 87, 062309 (2013).

[49] A. Wojcik, T. Luczak, P. Kurzynski, A. Grudka, T. Gdala, and M. Bednarska, Unmodulated spin chains as universal quantum wires, Phys. Rev. A 72, 034303 (2005).

[50] R. Yousefjani and A. Bayat, Simultaneous multiple-user quantum communication across a spin-chain channel, Phys. Rev. A 102, 012418 (2020).

[51] D. B. West, Introduction to Graph Theory, 2nd ed. (Prentice Hall, Upper Saddle River, 2001).

[52] A. S. Tanenbaum and T. Austin, Structured Computer Organization, 6th ed. (Pearson, Delhi, 2016).

[53] D. A. Patterson and J. L. Hennessy, Computer Organization and Design ARM Edition: The Hardware Software Interface (Morgan, Amsterdam, 2016).

[54] M. Gabbrielli and S. Martini, Programming Languages: Principles and Paradigms (Springer Science + Business Media, New York, 2010).

[55] C. P. Williams, Explorations in Quantum Computing (Springer Science + Business Media, New York, 2010).

[56] G. Brennen, E. Giacobino, and C. Simon, Focus on quantum memory, New J. Phys. 17, 050201 (2015).
[57] K. Heshami, D. G. England, P. C. Humphreys, P. J. Bustard, V. M. Acosta, J. Nunn, and B. J. Sussman, Quantum memories: Emerging applications and recent advances, J. Mod. Opt. 63, 2005 (2016).

[58] D. S. Dawoud and R. Peplow, Digital System Design: Use of Microcontroller (River, Gistrup, 2010), Vol. 2.

[59] D. Rosenberg, D. Kim, R. Das, D. Yost, S. Gustavsson, D. Hover, P. Krantz, A. Melville, L. Racz, G. O. Samach, S. J. Weber, F. Yan, J. L. Yoder, A. J. Kerman, and W. D. Oliver, 3D integrated superconducting qubits, npj Quantum Inf. 3, 42 (2017).

[60] V. Karimipour, M. S. Rad, and M. Asoudeh, Perfect quantum state transfer in two- and three-dimensional structures, Phys. Rev. A 85, 010302(R) (2012).

[61] S. S. Elder, C. S. Wang, P. Reinhold, C. T. Hann, K. S. Chou, B. J. Lester, S. Rosenblum, L. Frunzio, L. Jiang, and R. J. Schoelkopf, High-Fidelity Measurement of Qubits Encoded in Multilevel Superconducting Circuits, Phys. Rev. X 10, 011001 (2020).

[62] Y. Chen, C. Neill, P. Roushan, N. Leung, M. Fang, R. Barends, J. Kelly, B. Campbell, Z. Chen, B. Chiaro et al., Qubit Architecture with High Coherence and Fast Tunable Coupling, Phys. Rev. Lett. 113, 220502 (2014).

[63] F. Yan, P. Krantz, Y. Sung, M. Kjaergaard, D. L. Campbell, T. P. Orlando, S. Gustavsson, and W. D. Oliver, Tunable Coupling Scheme for Implementing High-Fidelity Two-Qubit Gates, Phys. Rev. Appl. 10, 054062 (2018).

[64] G. M. Reuther, D. Zueco, F. Deppe, E. Hoffmann, E. P. Menzel, T. Weiß1, M. Mariantoni, S. Kohler, A. Marx, E. Solano, R. Gross, and P. Hänggi, Two-resonator circuit quantum electrodynamics: Dissipative theory, Phys. Rev. B 81, 144510 (2010).

[65] Y. Sung, L. Ding, J. Braumüller, A. Vepsäläinen, B. Kannan, M. Kjaergaard, A. Greene, G. O. Samach, C. McNally, D. Kim, A. Melville, B. M. Niedzielski, M. E. Schwartz, J. L. Yoder, T. P. Orlando, S. Gustavsson, and W. D. Oliver, Realization of highfidelity $\mathrm{CZ}$ and ZZ-free iSWAP gates with a tunable coupler, arXiv:2011.01261.

[66] D. Zueco, G. M. Reuther, S. Kohler, and P. Hänggi, Qubitoscillator dynamics in the dispersive regime: Analytical theory beyond the rotating-wave approximation, Phys. Rev. A 80, 033846 (2009).

[67] M. D. Jenkins, D. Zueco, O. Roubeau, G. Aromí, J. Majer, and F. Luis, A scalable architecture for quantum computation with molecular nanomagnets, Dalton Trans. 45, 16682 (2016).

[68] M. Konstantinov, P. Petkov, P. Gancheva, V. Angelova, and I. Popchev, in Numerical Analysis and Its Applications, edited by L. Vulkov, J. Waśniewski, and P. Valamov, Lecture Notes in Computer Science Vol. 1196 (Springer, Berlin, 1996), pp. 258-265. 